\title{
Desarrollo y evaluación de alternativas tecnológicas para reducir el contenido de alcohol y el pH de vinos tintos
}

\author{
Development and evaluation of technological alternatives to reduce the alcohol content \\ and the $\mathrm{pH}$ of red wines.
}

Diego Piccardo ${ }^{1}$, Fernando Zamora ${ }^{2}$, Gustavo González-Neves ${ }^{3}$

Recibido: Febrero 2021

Aceptado: Marzo 2021

Resumen. - El objetivo de esta investigación fue desarrollar y evaluar tecnologías que permitan reducir el contenido de alcohol y el pH de los vinos tintos Pinot noir y Tannat sin disminuir su color y composición fenólica. Para reducir el contenido de alcohol y el pH se evaluó la sustitución parcial del mosto de uva madura por mosto de uva inmadura. Frente a un posible efecto de dilución al realizar la sustitución de mosto, se evaluó adicionalmente una maceración pre-fermentativa en caliente. La sustitución de mosto fue una técnica efectiva para reducir el contenido de alcohol y el $\mathrm{pH}$, sin modificar la concentración fenólica de los vinos elaborados en ambos cultivares. Adicionalmente, la maceración pre-fermentativa en caliente incrementó el color del vino y su concentración en polifenoles totales, antocianos, catequinas y proantocianidinas. El impacto de ambas técnicas estuvo determinado por las características varietales y las condiciones de maduración, por lo que es necesario continuar adaptando estas técnicas a diferentes cultivares y en otras situaciones de cultivo. El desarrollo de estas tecnologías es una herramienta interesante ya que pueden ser aplicadas de forma diferencial de acuerdo con el potencial enológico de la uva en cada vendimia, mitigando los efectos del cambio climático sobre su composición.

Palabras clave: Tannat; Pinot noir; maceración pre-fermentativa en caliente; color del vino; composición fenólica del vino.

Summary. - The objective of this research was to develop and evaluate technologies that reduce the alcohol content and pH of Pinot noir and Tannat red wines without reduce their color and phenolic composition. To reduce wine alcohol content and $\mathrm{pH}$, a partial substitution of the must of ripe grapes by must of unripe grapes was evaluated. Faced with a possible dilution effect when substituting must, a pre-fermentation hot maceration was additionally evaluated. Must substitution was an effective technique to reduce the alcohol content and the $\mathrm{pH}$ without modifying the phenolic concentration of wines made in both cultivars. Additionally, the pre-fermentation hot maceration increased the color of the wine and its concentration in total polyphenols, anthocyanins, catechins and proanthocyanins. The impact of both techniques was determined by the varietal characteristics and the ripening conditions, so it is necessary to continue adapting these techniques to different cultivars and in other growing situations. The development of these technologies is an interesting tool because they can be applied differentially according to the oenological potential of the grape in each harvest, mitigating the effects of climate change on its composition.

Keywords: Tannat; Pinot noir; pre-fermentative hot maceration; wine color; wine composition

\footnotetext{
${ }^{1}$ Dr en Ciencias Agrarias. Unidad de Tecnología de los Alimentos. Facultad de Agronomía. Udelar. Garzón 780, 12900. Montevideo. Uruguay. dpiccardo@fagro.edu.uy ORCID iD: https://orcid.org/0000-0002-1911-0686

${ }^{2}$ Dr en Ciencias Químicas. Departament de Bioquímica i Biotecnologia, Facultat d'Enologia. Universitat Rovira i Virgili, Tarragona, España fernando.zamora@urv.cat ORCID iD: https://orcid.org/0000-0002-4213-3528

3 Dr en Ciencias de los Alimentos. Unidad de Tecnología de los Alimentos. Facultad de Agronomía. Udelar. Garzón 780, 12900. Montevideo. Uruguay. gustavogn@fagro.edu.uy ORCID iD: https://orcid.org/0000-0002-0828-4752
} 
Introducción. - El color y el cuerpo del vino tinto son dos características sensoriales que determinan su calidad y su potencial para la crianza. Estas están asociados a la concentración de compuestos fenólicos del vino, principalmente antocianos y taninos, y a sus interacciones con otros compuestos durante la vinificación. Para elaborar vinos con cuerpo y de color intenso es necesario cosechar la uva en un estado avanzado de maduración y realizar una intensa extracción de los compuestos fenólicos durante la vinificación. La vinificación de uva con una madurez avanzada trae consigo dos problemas importantes en la elaboración de vinos. Por un lado, los vinos presentan altos valores de $\mathrm{pH}$ lo que ocasiona una menor efectividad del anhidrido sulfuroso y una mayor susceptibilidad al desarrollo de microorganismos indeseados, a la vez que puede ocasionar una disminución en la intensidad del color. En estas condiciones, se hace imprescindible la corrección del pH del vino, lo que resulta en un incremento en los costos de elaboración. Por otro lado, los vinos elaborados con uvas en madurez avanzada presentan alto contenido de alcohol. La aplicación de políticas públicas y la concientización por la salud del consumidor han llevado a una disminución del consumo de bebidas con altos niveles de alcohol. Si bien el consumo moderado de vino tinto puede tener efectos beneficiosos para la salud por su contenido significativo en compuestos bioactivos, el vino contiene valores de alcohol importantes, sobre todo cuando se elabora a partir de uvas cosechadas en estados avanzados de maduración.

Para reducir el contenido de alcohol y el $\mathrm{pH}$ del vino es posible cosechar las uvas en etapas tempranas de la maduración, con menor contenido de azúcares y $\mathrm{pH}$. Sin embargo, la inadecuada maduración de hollejos y semillas puede originar vinos mal coloreados, amargos, astringentes y herbáceos, afectando su calidad.

Una alternativa tecnológica para reducir el contenido del alcohol y el pH del vino, sin afectar su color y composición fenólica, puede ser la sustitución parcial del mosto obtenido a partir de la uva madura por mosto de uva inmadura. Esta técnica se basa en que los azúcares y ácidos, que determinan respectivamente el contenido de alcohol y el $\mathrm{pH}$ de los vinos, se encuentran localizados en la pulpa de la uva, en tanto que los compuestos fenólicos responsables del color y cuerpo del vino están localizados en los hollejos. Así, se logra macerar los hollejos y las semillas de la uva madura en un mosto sustituido parcialmente por mosto de uva inmadura, con menor concentración de azúcares y mayor acidez total. Sin embargo, existe una dificultad a la hora de predecir el efecto de esta técnica sobre la composición del vino, ya que es imposible cuantificar con exactitud cuanto mosto de uva inmadura se sustituye en relación con mosto total de la uva madura. Esto ocurre porque el mosto debe extraerse, luego del estrujado y despalillado de la uva, por decantación, sin ejercer presión sobre los hollejos y las semillas de la uva madura, para no extraer los compuestos fenólicos. De esta forma, un remanente de mosto queda retenido entorno a los hollejos y semillas. Por tanto, es posible que al realizar la sustitución del mosto pueda existir un efecto indeseado de dilución de los compuestos fenólicos afectando la calidad del vino. La maceración prefermentativa en caliente podría utilizarse como una técnica complementaria que permita potenciar la extracción de estos compuestos en etapas tempranas de la maceración, mitigando el efecto de dilución. La maceración pre-fermentativa en caliente consiste en calentar el mosto, previo a la fermentación alcohólica, a temperaturas comprendidas entre 40 y $80{ }^{\circ} \mathrm{C}$ por un tiempo variable y tiene como objetivo incrementar la extracción de compuestos fenólicos y el color del vino.

1. Objetivo. - El objetivo general de esta investigación fue determinar la efectividad de la sustitución del mosto de uva madura por mosto de uva inmadura y la maceración pre-fermentativa en caliente, para reducir el contenido de alcohol y el $\mathrm{pH}$ del vino, sin disminuir su color y su concentración fenólica. 


\section{Metodología. - .}

2.1. Vinificaciones y diseño experimental. - La investigación se llevó a cabo sobre uvas Tannat y Pinot noir cosechadas durante las vendimias 2016, 2017 y 2018. Ambos cultivares fueron cosechados manualmente en viñedos comerciales ubicados en el departamento de Canelones en el sur de Uruguay.

$\mathrm{Al}$ inicio del envero se cosecharon $100 \mathrm{~kg}$ de uva por variedad para obtener un mosto con alta acidez y baja concentración de azúcares. Las uvas se estrujaron, despalillaron (Alfa 60 R, Italcom, Piazzola Sul Brenta, Italia) y se prensaron ligeramente en una prensa manual para obtener $50 \mathrm{~L}$ de mosto de uva inmadura. El mosto de uva inmadura se sulfito con $100 \mathrm{mg} / \mathrm{L}$ de $\mathrm{K}_{2} \mathrm{~S}_{2} \mathrm{O}_{2}$, se desfangó, se envasó en un recipiente de $50 \mathrm{~L}$ y se conservó a $4{ }^{\circ} \mathrm{C}$ hasta su uso. Adicionalmente, cuando la uva alcanzó la madurez tecnológica, se cosecharon $120 \mathrm{~kg}$ de uva por variedad. Para cada variedad, la uva se distribuyó aleatoriamente en 12 lotes de $10 \mathrm{~kg}$. La uva se despalilló y estrujó y el mosto se sulfito con $100 \mathrm{mg} / \mathrm{L}$ de $\mathrm{K}_{2} \mathrm{~S}_{2} \mathrm{O}_{2}$ y se distribuyó en 12 recipientes de $10 \mathrm{~L}$ de capacidad. Los tanques con mosto se dividieron aleatoriamente en dos grupos de seis recipientes cada uno. Seis tanques mantuvieron el mosto original (mosto original - MO), mientras que en los otros seis tanques se sustituyó $3 \mathrm{~L}$ de mosto de uva original por $3 \mathrm{~L}$ de mosto de uva inmadura con el objetivo de reducir el contenido de azúcares y pH (mosto reducido en azúcares-MR).

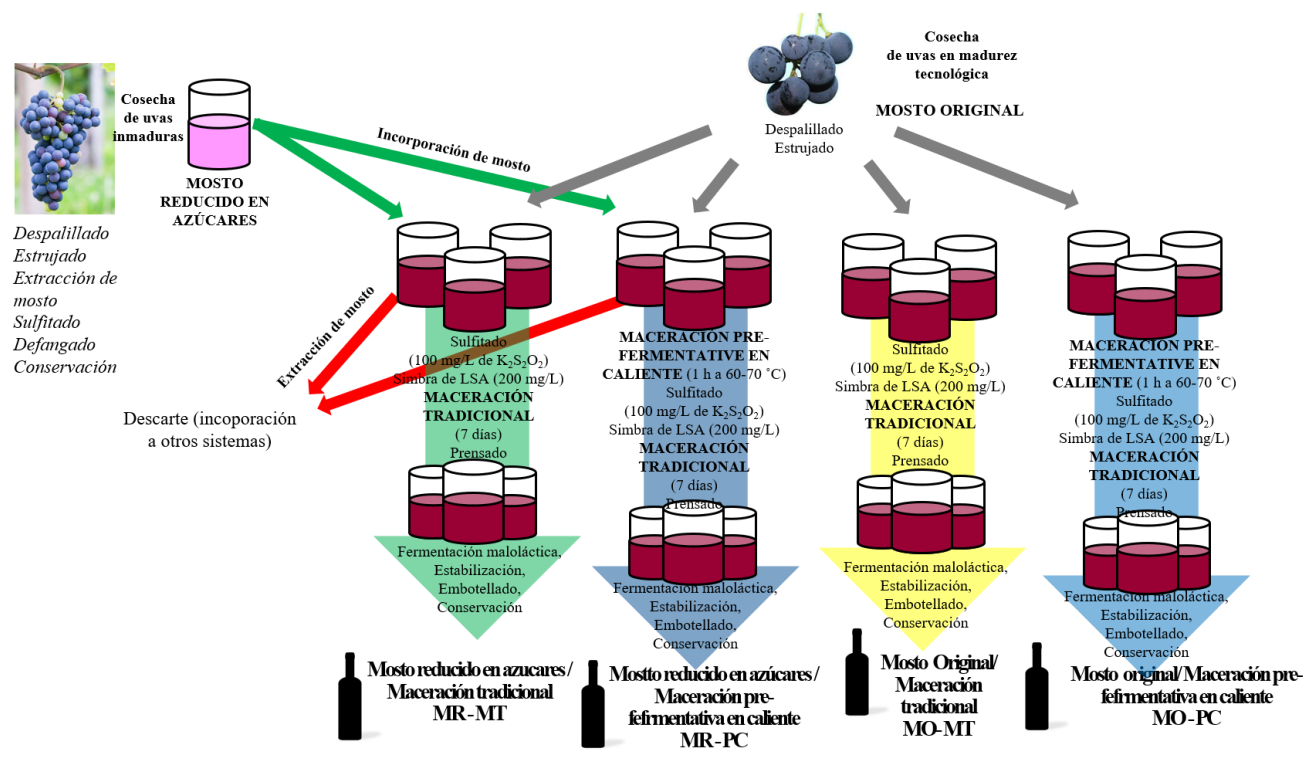

Figura I. - Vinificaciones y diseño experimental

A continuación, se maceraron tradicionalmente (MT) tres tanques de cada grupo experimental (MO y MR), mientras que los otros tres tanques se sometieron a maceración pre-fermentativa en caliente (PC) durante 1 hora a una temperatura entre 60 y $70{ }^{\circ} \mathrm{C}$. El calentamiento se realizó transfiriendo el orujo a tanques de acero inoxidable de $11 \mathrm{~L}$ que se sumergieron en un baño de agua caliente $\left(\mathrm{a} 80-90^{\circ} \mathrm{C}\right)$. Durante el calentamiento, el orujo se homogeneizó manualmente. Al finalizar el tratamiento térmico, los tanques de acero inoxidable se sumergieron en un baño de agua fría para refrigerarlos a temperatura ambiente (alrededor de $26^{\circ} \mathrm{C}$ ). Posteriormente, el mosto se trasladó a los tanques originales de $10 \mathrm{~L}$. Así, se obtuvieron cuatro grupos experimentales para cada cultivar: mosto original con maceración tradicional (MO-MT), mosto reducido en azúcares con maceración tradicional (MR-MT), mosto original con maceración pre-fermentativa en caliente (MO-PC), y 
mosto reducido en azúcares con maceración pre-fermentativa en caliente (MR-PC) (Figura 1).

Todos los tanques se inocularon con $200 \mathrm{mg} / \mathrm{L}$ de levadura seca activa (Saccharomyces cerevisiae ex bayanus Natuferm 804; Oenobiotech, París, Francia) y se fermentaron en contacto con hollejos y semillas. Durante la maceración, todos los contenedores se homogeneizaron manualmente una vez al día. Después de 7 días de maceración, se extrajo el vino de gota y se prensó ligeramente el orujo en una prensa manual. El vino de gota y el vino de prensa de cada tanque se unificaron y se mantuvieron en recipientes de $5 \mathrm{~L}$ a temperatura ambiente $\left(18 \pm 2^{\circ} \mathrm{C}\right)$. La fermentación alcohólica se completó cuando las medidas diarias de densidad del mosto fueron inferiores a $998 \mathrm{~g} / \mathrm{L}$ durante tres días consecutivos. Una vez finalizada la fermentación maloláctica espontánea, se estabilizaron con $100 \mathrm{mg} / \mathrm{L}$ de $\mathrm{K}_{2} \mathrm{~S}_{2} \mathrm{O}_{2}$ y $300 \mathrm{mg} / \mathrm{L}$ de lisozima (Delvo®Zyme, Delft, Holanda). Finalmente, los vinos se envasaron en botellas de $375 \mathrm{ml}$ de capacidad y se almacenaron en una cava oscura a temperatura ambiente de laboratorio hasta su análisis. Los análisis comenzaron 2 meses después del embotellado y finalizaron 3 semanas después.

2.2. Análisis estándares de mostos y vinos. - Se emplearon los métodos analíticos recomendados por la Organización Internacional de la Viña y el Vino [1] para determinar la concentración de azúcares, el pH y la acidez titulable de los mostos. El contenido de etanol, la acidez titulable y el $\mathrm{pH}$ de los vinos se analizaron utilizando un analizador de infrarrojos Winescan TM Autosampler 79,000 (Foss, EE. UU.) y el software Foss Integrator ver, 154 (Foss, Dinamarca).

2.3. Análisis espectrofotométricos de uvas y vinos. - La riqueza fenólica de la uva, su contenido en antocianos totales y su extractibilidad se determinó utilizando el procedimiento descrito por González-Neves et al. [2]. El color de los vinos se evaluó mediante índices espectrofotométricos clásicos. La intensidad colorante se determinó directamente sobre muestras de vino colocadas en una cubeta de $1 \mathrm{~mm}$ de paso óptico utilizando el método propuesto por Glories [3]. La composición fenólica de los vinos se evaluó mediante índices espectrofotométricos clásicos. Los polifenoles totales se determinaron mediante el método de Folin-Ciocalteu, según Singleton y Rossi [4]. La concentración de antocianos se analizó mediante la técnica descrita por Ribéreau-Gayon y Stonestreet [5]. Las catequinas se cuantificaron utilizando el método propuesto por Swain y Hillis [6], y las proantocianidinas se determinaron de acuerdo con Ribéreau-Gayon y Stonestreet [7].

2.4. Análisis estadístico. - Todos los resultados se expresan como el promedio aritmético \pm desviación estándar de tres repeticiones. El análisis de varianza multifactorial (MANOVA) se realizó con INFOSTAT [8], y se realizaron comparaciones múltiples entre muestras mediante el uso de la prueba de Hotelling.

\section{Resultados. -}

Composición del mosto en cosecha. - La Tabla 1 muestra la composición de los mostos Pinot noir y Tannat cosechados a principios del envero ( $1^{\text {er }}$ cosecha) y en madurez tecnológica ( $2^{\text {da }}$ cosecha) durante las vendimias 2016, 2017 y 2018. Las uvas cosechadas en la madurez tecnológica presentaron mayor concentración de azúcares y el pH y menor acidez titulable, indicando un mayor nivel de maduración. El potencial fenólico de la uva se determinó solamente sobre las cosechadas en madurez tecnológica que aportaron los hollejos y las semillas que fueron macerados durante la vinificación. Las uvas Tannat presentaron mayor riqueza fenólica y potencial en antocianos totales que las uvas Pinot Noir. Asimismo, el índice de extractabilidad de los antocianos fue mayor en las uvas Tannat, lo que indica que una menor proporción de antocianos son extraídos durante la maceración en relación con el potencial en antocianos totales. Estos resultados están de acuerdo 
con las características descritas para estas variedades en Uruguay [2].

\begin{tabular}{|c|c|c|c|c|c|c|c|c|}
\hline Cultivar & $\begin{array}{c}\text { Año de } \\
\text { vendimia }\end{array}$ & $\begin{array}{l}\text { Momento de } \\
\text { cosecha }\end{array}$ & $\begin{array}{c}\text { Azúcares } \\
(\mathrm{g} / \mathrm{L})\end{array}$ & $\begin{array}{c}\text { Acidez titulable } \\
\left(\mathrm{gH}_{2} \mathrm{SO}_{4} / \mathrm{L}\right)\end{array}$ & pH & A280 & ApH1 & EA (\%) \\
\hline \multirow{6}{*}{ Pinot Noir } & \multirow{2}{*}{2016} & $1^{e r} \operatorname{cosech} a$ & $129 \mathrm{~b}$ & $12.25 \mathrm{a}$ & $3.10 \mathrm{~b}$ & - & - & - \\
\hline & & $2^{d a} \operatorname{cosech} a$ & $243 \mathrm{a}$ & $3.62 \mathrm{~b}$ & $3.52 \mathrm{a}$ & 33.5 & 939 & 30.8 \\
\hline & \multirow{2}{*}{2017} & $1^{e r} \operatorname{cosech} a$ & $171 \mathrm{~b}$ & $5,11 \mathrm{a}$ & $3,29 \mathrm{~b}$ & - & - & - \\
\hline & & $2^{d a} \operatorname{cosech} a$ & $224 \mathrm{a}$ & $4,86 \mathrm{~b}$ & $3,45 \mathrm{a}$ & 53,4 & 212 & 26,0 \\
\hline & \multirow{2}{*}{2018} & $1^{e r}$ cosecha & $175 \mathrm{~b}$ & $4,48 \mathrm{a}$ & $3,22 \mathrm{~b}$ & - & - & - \\
\hline & & $2^{d a} \operatorname{cosech} a$ & $245 \mathrm{a}$ & $3,94 \mathrm{~b}$ & $3,59 \mathrm{a}$ & 58,9 & 434 & 25 \\
\hline \multirow{6}{*}{ Tannat } & \multirow{2}{*}{2016} & $1^{e r} \operatorname{cosech} a$ & $175 \mathrm{~b}$ & $8.43 \mathrm{a}$ & $3.12 \mathrm{~b}$ & - & - & - \\
\hline & & $2^{d a} \operatorname{cosech} a$ & $243 \mathrm{a}$ & $4.51 \mathrm{~b}$ & $3.31 \mathrm{a}$ & 47.5 & 2258 & 51.6 \\
\hline & \multirow{2}{*}{2017} & $1^{e r} \operatorname{cosech} a$ & $182 \mathrm{~b}$ & $5,98 \mathrm{a}$ & $3,10 \mathrm{~b}$ & - & - & - \\
\hline & & $2^{d a} \operatorname{cosech} a$ & $193 \mathrm{a}$ & $5,48 \mathrm{~b}$ & $3,23 \mathrm{a}$ & 61,5 & 949 & 49,2 \\
\hline & \multirow{2}{*}{2018} & $1^{e r} \operatorname{cosech} a$ & $191 \mathrm{~b}$ & $4,19 \mathrm{a}$ & $3,14 \mathrm{~b}$ & - & - & - \\
\hline & & $2^{d a} \operatorname{cosech} a$ & $263 \mathrm{a}$ & $3,63 \mathrm{~b}$ & $3,27 \mathrm{a}$ & 81,6 & 2334 & 47 \\
\hline
\end{tabular}

Tabla 1. - Composición fenólica de los vinos según cultivar y año de vendimia. Medias con distinta letra indican diferencias significativas $(\mathrm{p}<0.05)$. A280: riqueza fenólica de la uva, ApH1: potencial en antocianos totales, EA

(\%): es índice de extractibilidad de los antocianos.

El potencial enológico de las uvas fue diferente de acuerdo con el año de vendimia. Tanto las uvas Tannat como Pinot noir presentaron mayor concentración de azúcares y riqueza fenólica, y menor índice de extractibilidad de los antocianos en la vendimia 2018. El potencial en antocianos totales de las uvas Pinot noir fue mayor en la vendimia 2016, en tanto que en las uvas Tannat las mayores concentraciones se registraron en las vendimias 2016 y 2018 respectivamente. Para ambos cultivares, el menor potencial enológico de las uvas se registró en la vendimia 2017. Ferrer et al. [9] demostraron que los compuestos relacionados con la calidad de la uva se ven favorecidos por la acumulación de temperaturas durante las primeras etapas del ciclo de cultivo, mientras que las altas temperaturas y la disponibilidad hídrica durante la maduración los afectan negativamente. Estas condiciones son muy variables entre años, determinando diferencias en el potencial enológico de las uvas entre vendimias. Se debe considerar que las uvas Pinot noir presentan un ciclo de maduración menor respecto a las uvas Tannat, por lo que las condiciones climáticas durante este período son diferentes. Además, ambos cultivares presentan un potencial enológico diferente. Las uvas Tannat se caracterizan por tener contenidos muy elevados de pigmentos (antocianos) y taninos, en tanto que las uvas Pinot noir presentan una gran riqueza polifenólica (sobre todo en semillas), constituida casi exclusivamente por taninos, con muy bajos contenidos de antocianos.

3.1. Efectos sobre el contenido de etanol y el pH. - En la Tabla 2 se observan los efectos del año de vendimia, la composición del mosto y la técnica de maceración sobre el contenido de alcohol, la acidez total y el $\mathrm{pH}$ de los vinos.

El año de vendimia expresa el contenido promedio de alcohol, la acidez titulable y el $\mathrm{pH}$ de todos los vinos producidos en la misma cosecha, independientemente de la composición del mosto y la técnica de maceración. Los vinos Pinot noir y Tannat producidos a partir de la cosecha 2018 y 2016 presentaron mayor contenido de alcohol respecto a los elaborados en la vendimia 2017. Los valores más altos de $\mathrm{pH}$ se registraron en los vinos producidos en 2017 y 2018 para Pinot noir y 2016 y 2018 para Tannat respectivamente. De acuerdo con los resultados presentados en la Tabla 1, las uvas Pinot noir y Tannat recolectadas en 2016 y 2018 tuvieron mejores condiciones de maduración lo que permitió cosecharlas con un mayor nivel de madurez, con mayores concentraciones de azúcares, respecto a las cosechadas en el 2017. 
La composición del mosto expresa el contenido promedio de alcohol, la acidez titulable y el $\mathrm{pH}$ de todos los vinos producidos con mosto original (MO) o mosto reducido en azúcares (MR), independientemente del año de vendimia y la técnica de maceración. Los vinos MR de ambos cultivares presentaron menor contenido de etanol y $\mathrm{pH}$ y mayor acidez titulable que los vinos MO. El reemplazo del mosto de las uvas maduras por el mosto de las uvas inmaduras implicaba una disminución en el contenido de azúcares y pH y un aumento de la acidez titulable [12,13). Esta técnica se realizó previo a la maceración fermentativa, lo que implicó que las levaduras inoculadas tuvieran una menor concentración de azúcares para transformar en alcohol, explicando el menor contenido de éste en los vinos. Asimismo, los resultados obtenidos en la acidez titulable y el pH de los vinos MR de ambos cultivares se corresponden con la composición del mosto de uva inmadura utilizado en la sustitución (Tabla 1).

La técnica de maceración expresa el contenido promedio de alcohol, la acidez titulable y el $\mathrm{pH}$ de todos los vinos producidos por la maceración tradicional (MT) o la maceración prefermentativa en caliente (PC), independientemente del año de vendimia y la composición inicial del mosto. No se encontraron diferencias significativas debidas a la técnica de maceración, en la composición básica de los vinos de ambos cultivares, excepto por un leve incremento en el contenido de alcohol de los vinos PC elaborados a partir del cultivar Tannat. Investigaciones previas $[10,11]$ reportaron un mayor contenido de alcohol en los vinos Tannat elaborados por maceración pre-fermentativa en caliente debido a una leve evaporación del agua durante la etapa de calentamiento que podría originar una concentración del mosto y a una mayor extracción de compuestos nitrogenados que podría contribuir a mejorar la eficiencia fermentativa de las levaduras. Además, reportaron que los vinos elaborados por esta técnica de maceración podrían presentar mayor $\mathrm{pH}$, ya que, durante el calentamiento pre-fermentativo, aumenta la extracción de cationes, lo que resulta en un aumento en el pH dado por la salificación del ácido tartárico.

\begin{tabular}{|c|c|c|c|c|c|}
\hline Variedad & Factor analiza & & Alcohol (\% v/v) & $\begin{array}{c}\text { Acidez titulable } \\
\left.\text { (g/L } \mathrm{H}_{2} \mathrm{SO}_{4}\right)\end{array}$ & pH \\
\hline \multirow{11}{*}{ Pinot noir } & \multirow{3}{*}{ Año de vendimia } & 2016 & $13,0 \mathrm{~b}$ & $4,23 \mathrm{a}$ & $3,61 \mathrm{c}$ \\
\hline & & 2017 & $12,6 \mathrm{c}$ & $2,51 \mathrm{c}$ & $3,87 \mathrm{a}$ \\
\hline & & 2018 & $14,7 \mathrm{a}$ & $3,27 \mathrm{c}$ & $3,80 \mathrm{~b}$ \\
\hline & \multirow{2}{*}{$\begin{array}{l}\text { Compisición inicial del } \\
\text { mosto }\end{array}$} & MO & 14,3 a & $2,93 \mathrm{~b}$ & 3,87 a \\
\hline & & MR & $12,6 \mathrm{~b}$ & $3,74 \mathrm{a}$ & $3,65 \mathrm{~b}$ \\
\hline & \multirow{2}{*}{ Tecnica de maceración } & MT & $13,4 \mathrm{a}$ & 3,31 a & $3,75 \mathrm{a}$ \\
\hline & & $\mathrm{PC}$ & $13,4 \mathrm{a}$ & $3,36 \mathrm{a}$ & $3,77 \mathrm{a}$ \\
\hline & \multirow{4}{*}{$\begin{array}{c}\text { Composición inicial de } \\
\text { mosto X Técnica de } \\
\text { maceración }\end{array}$} & MO-MT & $14,4 \mathrm{a}$ & $2,93 \mathrm{~b}$ & $3,86 \mathrm{a}$ \\
\hline & & MR-MT & $12,5 \mathrm{c}$ & 3,79 a & $3,63 \mathrm{~b}$ \\
\hline & & MO-PC & $14,2 \mathrm{~b}$ & $2,92 \mathrm{~b}$ & $3,87 \mathrm{a}$ \\
\hline & & MR-PC & $12,6 \mathrm{c}$ & $3,70 \mathrm{a}$ & $3,63 \mathrm{~b}$ \\
\hline \multirow{11}{*}{ Tannat } & \multirow{3}{*}{ Año de vendimia } & 2016 & $14.0 \mathrm{~b}$ & $4.30 \mathrm{a}$ & $3.92 \mathrm{a}$ \\
\hline & & 2017 & $11.2 \mathrm{c}$ & $2.93 \mathrm{c}$ & $3.86 \mathrm{c}$ \\
\hline & & 2018 & $15.4 \mathrm{a}$ & $3.85 \mathrm{~b}$ & $3.89 \mathrm{~b}$ \\
\hline & \multirow{2}{*}{ Composición de mosto } & MO & $14.0 \mathrm{a}$ & $3.51 \mathrm{~b}$ & $3.95 \mathrm{a}$ \\
\hline & & MR & $13.0 \mathrm{~b}$ & $3.88 \mathrm{a}$ & $3.83 \mathrm{~b}$ \\
\hline & \multirow{2}{*}{ Tecnica de maceración } & MT & $13.3 \mathrm{~b}$ & $3.74 \mathrm{a}$ & $3.87 \mathrm{a}$ \\
\hline & & $\mathrm{PC}$ & $13.7 \mathrm{a}$ & $3.64 \mathrm{a}$ & $3.92 \mathrm{a}$ \\
\hline & \multirow{4}{*}{$\begin{array}{l}\text { Composición de mosto - } \\
\text { Técnica de maceración }\end{array}$} & MO-MT & $14.0 \mathrm{a}$ & $3.61 \mathrm{~b}$ & $3.92 \mathrm{~b}$ \\
\hline & & MR-MT & $12.6 \mathrm{c}$ & $3.87 \mathrm{a}$ & $3.81 \mathrm{~d}$ \\
\hline & & MO-PC & $14.0 \mathrm{a}$ & $3.40 \mathrm{c}$ & $3.98 \mathrm{a}$ \\
\hline & & MR-PC & $13.4 \mathrm{~b}$ & $3.88 \mathrm{a}$ & $3.85 \mathrm{c}$ \\
\hline
\end{tabular}

Tabla 2. - Composición básica de los vinos según cultivar. Medias con distinta letra indican diferencias 
significativas $(\mathrm{p}<0.05)$. MO: Mosto original. MR: Mosto reducido en azúcares. MT, Maceración tradicional. PC: Maceración pre-fermentativa en caliente.

La interacción entre la composición del mosto y la técnica de maceración expresa el contenido promedio de alcohol y $\mathrm{pH}$ de todos los vinos producidos con mosto original y la maceración tradicional (MO-MT), mosto reducido en azúcares y maceración tradicional (MR-MT), mosto original y maceración pre-fermentativa en caliente (MO-PC), o mosto reducido en azúcares y maceración pre-fermentativa en caliente (MR-PC), independientemente de la cosecha. Para ambos cultivares, el contenido de alcohol y $\mathrm{pH}$ de los vinos MO-MT y MO-PC fue significativamente mayor que el de los vinos MR-MT y MR-PC. Estos resultados indican que la sustitución es una técnica efectiva para elaborar vinos con menores contenido de alcohol y $\mathrm{pH}$. Resultados similares fueron reportados por Kontoudakis et al. [12] y Rolle et al. [13], empleando técnicas de sustitución de mostos similares.

3.2. Composición fenólica del vino. - La composición fenólica de los vinos fue diferente según el año de vendimia (Tabla 3). Los vinos Pinot noir producidos en 2016 presentaron mayores concentraciones de polifenoles, antocianos, catequinas y proantocianidinas. Por su parte, los vinos Tannat 2016 presentaron mayor concentración de polifenoles y proantocianidinas, en tanto que los vinos 2018 presentaron mayores concentraciones de antocianos y catequinas. Para ambos cultivares los vinos producidos en la vendimia 2017 presentaron las menores concentraciones. El nivel de maduración de las uvas cosechadas en la segunda fecha (Tabla 1) determinó fuertemente la composición fenólica de los vinos.

La composición inicial del mosto no modifico la concentración de polifenoles, antocianos, catequinas y proantocianidinas de los vinos de ambos cultivares. Otras técnicas propuestas para reducir el contenido de alcohol del vino han afectado de manera selectiva la concentración de las distintas familias fenólicas [13]. Según estos autores, la concentración más baja de etanol podría afectar la extracción de taninos altamente polimerizados de las uvas durante la fermentación. Además, sugieren que, si bien se esperaría una menor concentración de antocianos, debido al remplazo de una parte del mosto, esto no implica una pérdida, ya que este reemplazo se realizó previo a la maceración. Kontoudakis et al. [12] encontraron que los antocianos no son afectados cuando se reduce la concentración de etanol en un $3.0 \% \mathrm{v} / \mathrm{v}$ por un reemplazo de una parte del mosto de uva madura por vino con bajo contenido de alcohol en tanto que las proantocianidinas eran menos abundantes. 


\begin{tabular}{|c|c|c|c|c|c|c|}
\hline Variedad & Factor analizado & & $\begin{array}{c}\text { Polifenoles } \\
\text { Totales }(\mathrm{mg} / \mathrm{L})\end{array}$ & $\begin{array}{l}\text { Antocianos } \\
(\mathrm{mg} / \mathrm{L})\end{array}$ & $\begin{array}{c}\text { Catequinas } \\
(\mathrm{mg} / \mathrm{L})\end{array}$ & $\begin{array}{c}\text { Proantocianidinas } \\
(\mathrm{mg} / \mathrm{L})\end{array}$ \\
\hline \multirow{11}{*}{ Pinot noir } & \multirow{3}{*}{ Año de vendimia } & 2016 & $1641 \mathrm{a}$ & $350 \mathrm{a}$ & $1528 \mathrm{a}$ & $3045 \mathrm{a}$ \\
\hline & & 2017 & $998 \mathrm{c}$ & $213 \mathrm{c}$ & $379 \mathrm{c}$ & $1264 \mathrm{c}$ \\
\hline & & 2018 & $1182 \mathrm{~b}$ & $243 \mathrm{~b}$ & $774 \mathrm{~b}$ & $1667 \mathrm{~b}$ \\
\hline & \multirow{2}{*}{$\begin{array}{l}\text { Compisición inicial del } \\
\text { mosto }\end{array}$} & MO & $1254 \mathrm{a}$ & $269 \mathrm{a}$ & $884 \mathrm{a}$ & 1990 a \\
\hline & & MR & $1281 \mathrm{a}$ & $268 \mathrm{a}$ & 902 a & $1994 \mathrm{a}$ \\
\hline & \multirow{2}{*}{ Tecnica de maceración } & MT & $900 \mathrm{~b}$ & $218 \mathrm{~b}$ & $517 \mathrm{~b}$ & $1112 \mathrm{~b}$ \\
\hline & & $\mathrm{PC}$ & $1635 \mathrm{a}$ & $319 \mathrm{a}$ & $1269 \mathrm{a}$ & $2872 \mathrm{a}$ \\
\hline & \multirow{4}{*}{$\begin{array}{l}\text { Composición inicial de } \\
\text { mosto X Técnica de } \\
\text { maceración }\end{array}$} & MO-MT & $908 \mathrm{c}$ & $214 \mathrm{~b}$ & $487 \mathrm{~b}$ & $1081 \mathrm{~b}$ \\
\hline & & MR-MT & $892 \mathrm{c}$ & $222 \mathrm{~b}$ & $548 \mathrm{~b}$ & $1144 \mathrm{~b}$ \\
\hline & & MO-PC & $1599 \mathrm{~b}$ & $325 \mathrm{a}$ & $1280 \mathrm{a}$ & 2899 a \\
\hline & & MR-PC & $1671 \mathrm{a}$ & $313 \mathrm{a}$ & $1258 \mathrm{a}$ & $2845 \mathrm{a}$ \\
\hline \multirow{11}{*}{ Tannat } & \multirow{3}{*}{ Año de vendimia } & 2016 & $2479 \mathrm{a}$ & $1052 \mathrm{a}$ & $1769 \mathrm{~b}$ & $4172 \mathrm{a}$ \\
\hline & & 2017 & $1624 \mathrm{c}$ & $614 \mathrm{~b}$ & $1420 \mathrm{c}$ & $2690 \mathrm{c}$ \\
\hline & & 2018 & $2140 \mathrm{~b}$ & $1165 \mathrm{a}$ & $1883 \mathrm{a}$ & $3260 \mathrm{~b}$ \\
\hline & \multirow{2}{*}{ Composición de mosto } & MO & $2045 \mathrm{a}$ & $960 \mathrm{a}$ & $1667 \mathrm{a}$ & $3397 \mathrm{a}$ \\
\hline & & MR & $2117 \mathrm{a}$ & $994 \mathrm{a}$ & $1714 \mathrm{a}$ & $3352 \mathrm{a}$ \\
\hline & \multirow{2}{*}{ Tecnica de maceración } & MT & $1784 \mathrm{~b}$ & $838 \mathrm{~b}$ & $1281 \mathrm{~b}$ & $2764 \mathrm{~b}$ \\
\hline & & $\mathrm{PC}$ & 2379 a & $1117 \mathrm{a}$ & $2100 \mathrm{a}$ & $3985 \mathrm{a}$ \\
\hline & \multirow{4}{*}{$\begin{array}{l}\text { Composición de mosto - } \\
\text { Técnica de maceración }\end{array}$} & MO-MT & $1821 \mathrm{c}$ & $832 \mathrm{c}$ & $1273 \mathrm{~b}$ & $2792 \mathrm{~b}$ \\
\hline & & MR-MT & $1747 \mathrm{~d}$ & $843 \mathrm{c}$ & $1289 \mathrm{~b}$ & $2735 \mathrm{~b}$ \\
\hline & & MO-PC & $2345 \mathrm{~b}$ & $1088 \mathrm{~b}$ & $2061 \mathrm{a}$ & $4001 \mathrm{a}$ \\
\hline & & MR-PC & $2413 \mathrm{a}$ & $1146 \mathrm{a}$ & $2141 \mathrm{a}$ & $3968 \mathrm{a}$ \\
\hline
\end{tabular}

Tabla 3. - Composición fenólica de los vinos según cultivar. Medias con distinta letra indican diferencias significativas $(\mathrm{p}<0.05)$. MO: Mosto original. MR: Mosto reducido en azúcares. MT, Maceración tradicional. PC: Maceración pre-fermentativa en caliente.

Las concentraciones de polifenoles, antocianos, catequinas y proantocianidinas de los vinos PC fueron significativamente más altos que los de los vinos MT en ambos cultivares. Estos resultados concuerdan con estudios previos $[10,11]$ y confirman que esta técnica permite incrementar la extracción de polifenoles, ya que el calentamiento pre-fermentativo contribuye a degradar los tejidos de los hollejos, liberando estos compuestos en el mosto.

$\mathrm{Al}$ analizar el efecto conjunto de la composición del mosto y la técnica de maceración, se observa que los vinos MO-PC y MR-PC presentaron mayores contenidos de polifenoles, antocianos, catequinas y proantocianidinas que los vinos MO-MT y MR-MT. Los vinos Pinot noir elaborados con las técnicas de reemplazo de mosto y maceración pre-fermentativa en caliente (MRPC) presentaron mayor concentración de polifenoles totales en tanto que, los vinos Tannat elaborados con ambas técnicas de vinificación presentaron mayor concentración de polifenoles totales y antocianos. Esta respuesta diferencial está determinada por el potencial enológico del cultivar y las condiciones de maduración de las uvas al momento de cosecha.

3.3. Color del vino. - La intensidad colorante de los vinos Pinot noir y Tannat se observa en la Figura II. Los vinos Pinot noir con mayor intensidad colorante fueron elaborados a partir de la vendimia 2016 en tanto que los elaborados en la vendimia 2017 y 2018 presentaron valores significativamente menores. Los vinos Tannat mostraron mayor intensidad colorante que los vinos Pinot noir, siendo significativamente más alta en los elaborados en la vendimia 2016. El color del vino está determinado por la concentración de antocianos, su extracción durante la maceración y las interacciones con otros compuestos durante la vinificación y conservación del vino. Asimismo, se debe considerar las características varietales. Los vinos Pinot noir presentan baja concentración de antocianos, por lo que son menos estables. Los vinos Tannat se caracteriza por presentar una alta concentración de antocianos, pero difíciles de extraer durante la vinificación. 
a - Pinot noir

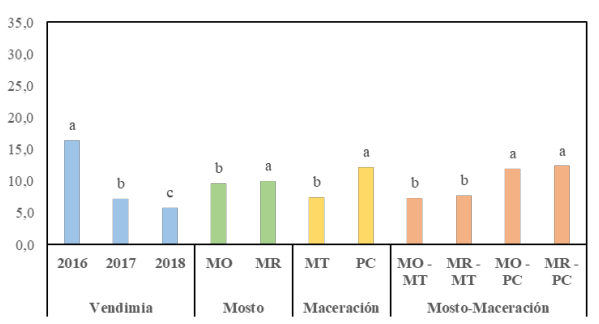

b - Tannat

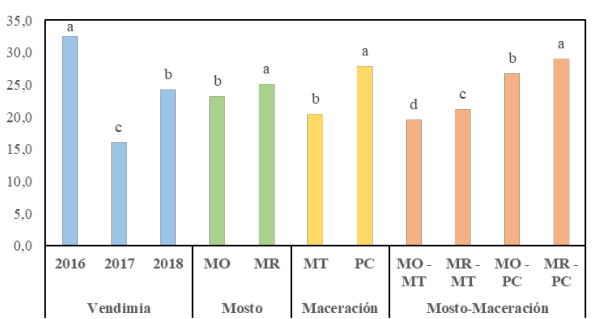

Figura II. - Intensidad colorante de los vinos Pinot noir y Tannat

Para ambos cultivares, los vinos MR presentaron mayor intensidad colorante. Este resultado se debe al efecto del $\mathrm{pH}$ sobre el equilibrio de las distintas formas antociánicas. Cuando el $\mathrm{pH}$ disminuye, el equilibrio entre las diferentes formas de los antocianos se desplaza hacia la forma roja de las moléculas, el catión flavilio [14].

Los vinos PC de ambos cultivares presentaron mayor intensidad colorante en relación con los vinos MT. Este resultado se debe a la mayor extracción de antocianos durante la etapa prefermentativa y ha sido reportado previamente en otras investigaciones [10,11].

Al analizar el efecto de la combinación de ambas técnicas de vinificación, se observó que los vinos Pinot noir MO-PC y MR-PC presentaron la mayor intensidad de color sin diferenciarse significativamente entre sí. Este incremento en la intensidad colorante está directamente relacionado a una mayor concentración de antocianos extraídos durante la maceración prefermentativa en caliente. Adicionalmente, en los vinos Tannat se observó un incremento en la intensidad colorante dado por la combinación de ambas técnicas de vinificación, por un lado, el mayor valor de $\mathrm{pH}$ que modifica el equilibrio de los antocianos hacia formas menos coloreadas $\mathrm{y}$, por otro la mayor extracción de antocianos por la maceración pre-fermentativa en caliente.

4. Conclusiones. - La sustitución del mosto de uva madura por mosto de uva inmadura y la maceración pre-fermentativa en caliente son alternativas tecnológicas que permiten reducir el contenido de alcohol y el pH, e incrementar la concentración de las distintas familias fenólicas y el color de los vinos tintos de ambos cultivares. La técnica propuesta de sustitución de mosto fue efectiva para reducir el contenido de alcohol y el pH del vino y no modificó su composición fenólica. La maceración pre-fermentativa en caliente permitió incrementar el color del vino y su concentración en polifenoles, antocianos, catequinas y proantocianidinas.

El impacto de ambas técnicas de vinificación estuvo determinado por las características varietales y las condiciones de maduración, por lo que es necesario continuar la investigación adaptando estas técnicas a distintos cultivares y en otras situaciones de cultivo. El desarrollo de estas tecnologías es una herramienta interesante ya que pueden ser aplicadas de forma diferencial de acuerdo con el potencial enológico de la uva en cada vendimia, mitigando los efectos del cambio climático sobre su composición.

5. Agradecimientos. - D. Piccardo agradece el apoyo financiero de CAP (Comisión Académica de Posgrado de la Universidad de la República), ANII (Agencia Nacional de Investigación e Innovación de Uruguay; beca MOV_CA_2015_1_107599), CSIC (Comisión Sectorial de Investigación Científica de la Universidad de la República; becas Movilidad 2017 y 2018).

Los autores agradecen a Dr. Ing Agr. Guzmán Favre, Dra. Ing. Alim. Olga Pascual y Dr. Joan Miquel Canals por su participación y apoyo en las distintas etapas de la tesis doctoral. También agradecen al INAVI (Instituto Nacional de Vitivinicultura de Uruguay) por el apoyo técnico, y a Establecimiento Juanicó y Bodega Olga Silva por las uvas empleadas en los ensayos. 


\section{Referencias}

[1] OIV. Compendium of international: methods of analysis of wines and musts. Vol 1. 2018. Organisation Internationale de la Vigne et du Vin. Paris, France.

[2] G. González-Neves, D. Charamelo, J. Balado, L. Barreiro, R. Bochicchio, G. Gatto, G. Gil, A. Tesore, A. Carbonneau, M. Moutounet. Phenolic potential of Tannat, Cabernet-Sauvignon and Merlot grapes and their correspondence with wine composition. Analytical Chimca Acta, 2004. 513:191-196

[3] Y. Glories. La couleur des vins rouges. 2e. Partie : Mesure, origine et interprétation. Connaissance Vigne Vin, 1984. 18 (4): 253-271.

[4] V. Singleton, J. Rossi. Colorimetry of total phenolics with phosphomolybdic and phosphotungstic acid reagents. American Journal of Enology and Viticulture, 1965. 16, 144 158.

[5] P. Ribéreau-Gayon, E. Stonestreet. Le dosage des anthocyanes dans les vins rouges. Bulletin de la Société Chimique de France, 1965. 9, 2649-2653.

[6] T. Swain, W. Hillis. The phenolic constituents of Prunus domestica. I. The quantitative analysis of phenolic constituents. Journal of Science and Food Agriculture, 1959. 10: 63-68.

[7] P. Ribéreau-Gayon, E. Stonestreet. Dosage des tanins dans du vin rouge et détermination de leur structure. Chimie Analytique, 1966. 48, 188-196.

[8] J.A. Di Rienzo, F. Casanoves, M.G.Balzarini, L. Gonzalez, M. Tablada, C.W Robledo. InfoStat versión 2015. Grupo InfoStat, FCA, Universidad Nacional de Córdoba, Argentina. URL http://www.infostat.com.ar

[9] M. Ferrer, G. Echeverría, M. Miras-Avalos. Meteorological Conditions: Influence on Yield, Sanitary Status and Grape Composition. International Journal of Environmental \& Agriculture Research, 2017.8 (3): 16-27.

[10]D. Piccardo D, G. Favre, O. Pascual, J.M. Canals, F. Zamora, G. Gonzalez. Influence of the use of unripe grapes to reduce ethanol content and $\mathrm{pH}$ on the color, polyphenol and polysaccharide composition of conventional and hot macerated Pinot Noir and Tannat wines. European Food Research and Technology, 2019. 245 (6): 1321-1335.

[11]D. Piccardo, Evaluación de distintas alternativas en la elaboración de vinos tintos de la variedad Tannat. Tesis de Maestría. 2015. Montevideo, Facultad de Agronomía, Uruguay.

[12] N. Kontoudakis, M. Esteruelas, F. Fort, J.M. Canals, V. De Freitas, F. Zamora. Influence of the heterogeneity of grape phenolic maturity on wine composition and quality. Food Chemestry, 2011. 124: 67-774.

[13]L. Rolle, V. Englezos, F. Torchio, F. Cravero, S. Río Segade, K. Rantsiou, S. Giacosa, A. Gambuti, V. Gerbi, L. Cocolin. Alcohol reduction in red wines by technological and microbiological approaches: A comparative study. Australian Journal of Grape Wine Research, 2018. 24 (1): 62-74.

[14]F. He, N.N. Liang, L. Mu, Q.H. Pan, J. Wang, M.J. Reeves, C.Q. Duan. Anthocyanins and their variation in red wines I. Monomeric anthocyanins and their color expression. Molecules, 2012. 17:1571-1601 